\title{
Stroke Prognosis through Retinal Image Analysis
}

\author{
${ }^{1}$ Jeena R S, ${ }^{2} \mathrm{Dr}$. Sukesh Kumar A \\ ${ }^{1}$ College of Engineering Trivandrum ${ }^{2}$ RajivGandhi Institute of development Studies, Trivandrum \\ jeena_rs@yahoo.com,drsukeshkumar@yahoo.in
}

\begin{abstract}
Many eye diseases as well as systemic diseases usually used to manifest in the retina. The innovations in the field of retinal imaging have paved the way to the development of tools for assisting physicians in stroke prognosis. Stroke is one of the leading causes of adult disability in most of the developing countries. Diagnosis of stroke during the initial stage is crucial for timely prevention and cure. Retinal imaging provides a non invasive technique of predicting the possibility of stroke. This research work focuses on the prediction of retinal ischemia from retinal fundus images and thereby predicting the occurance of stroke. Preprocessing of retinal images is done by retinex processing and morphological operations are done to remove noisy background. Branching points are detected and various features like major axis length, mean diameter, orientation, eccentricity, fractal dimension and tortuosity for the branching blood vessels are computed. This has been compared for various diseases like diabetic retinopathy, hypertensive retinopathy and retinal ischemia against a set of healthy retinal images. Classification has been implemented by Artificial Neural Networks which gives an accuracy of $89 \%$ and the results proved to be promising.
\end{abstract}

Keywords: Stroke, prognosis, retinal image, retinex, retinal ischemia

\section{Introduction}

Stroke is a form of cardiovascular disease affecting the blood supply to the brain. It remains as a leading cause of disability and death for people of all races and ethnicities [1]. Stroke is a physical condition that occurs due to insufficient supply of blood to the brain cells. This damages the brain cells ultimately leading to their death. A clot in the blood vessel or a blood vessel rupture can interrupt the blood supply to brain. Stroke can be subdivided into two types : ischemic and hemorrhagic. Ischemic stroke accounts for almost $85 \%$ of the cases. It occurs as a result of a block within a blood vessel supplying blood to the brain. Hemorrhagic stroke occurs when a weakened blood vessel ruptures. When an obstruction occurs within a blood vessel supplying blood to brain, the vessels carrying blood to eye will also be affected during the initial stages. This is termed as Retinal ischemia. Persons suffering from Retinal ischemia are more prone to stroke.

The retina can be viewed and analyzed using non-invasive in vivo functional techniques. Retinal imaging and image analysis have developed rapidly over the past ten years, and image analysis plays an important role in the care of patients with retinal diseases, as well as diseases that manifest in the retina. Retinal imaging allows diagnosis of various eye diseases as well as the prognosis of other complications of diabetes, hypertension and cardiovascular diseases like stroke. The retina is a layered tissue lining the inner part of eye that enables the conversion of incoming light into a neural signal that is appropriate for further processing in the brain. It is therefore an extension of human brain. The 
Jeena R S, Dr. Sukesh Kumar A; Stroke Prognosis through Retinal Image Analysis. Advances in Image and Video Processing, Volume 5 No 2, April (2017); pp: 13-21

ability to image the retina and develop methods for analyzing the images is of great interest. The ocular structures of retina have to be optically transparent for image formation. Thus, with suitable methods, the retina is visible from the outside, making the retinal tissue, and thereby the tissue of brain, accessible for imaging non invasively. Research works show that microvasculature of retina and brain is closely linked in terms of anatomy and physiology [2].

Cardiovascular disease manifests itself in the retina different ways. Hypertension and atherosclerosis cause changes in the ratio between the diameter of retinal arteries and veins, Thinning of the arteries and widening of the veins is associated with an increased risk of stroke. Retinal arterioles share similar anatomical, physiological and embryological characteristics with cerebral arterioles. Researchers have suggested that microaneurysms or arteriolar narrowing seen in the retinal circulation are risk factors for cerebrovascular disease such as stroke.[2]Hypertension can also cause direct retinal ischemia, which causes retinal infarcts visible as cotton wool spots and choroidal infarcts visible as deep retinal white spots. Also, systemic vascular disease can cause arterial and venous occlusions, known as central and branch arterial occlusions and central and branch venous occlusions. Morphological changes in blood vessel shape, branching pattern, width, tortuosity, appearance of retinal lesions, branching angle, branching coefficient and fractal dimension are some of the abnormalities in vascular pattern of retina associated with cardiovascular diseases like stroke.

\section{Literature survey}

The idea of Retinex was conceived by Edwin Land [3] as a model of lightness and color perception of the human vision. Edwin Land coined word for his model of human color vision, combining the retina of the eye and the cerebral cortex of the brain. The Retinex is a human-perception based image processing algorithm which provides color constancy and dynamic range compression.

Daniel J. Jobson et al. has proposed multiscale retinex [4], which fills the gap between color images and the human observation of scenes. The enhanced image has good dynamic range compression and color constancy but this technique fails to give good color rendition.

Youhei Terai et al. [5] proposed a retinex model for color image contrast enhancement. The luminance signal is processed to reduce the computation time without changing color components. The algorithm performs better for gray images rather than color images.A color image enhancement algorithm based on human visual system based on adaptive filter is proposed by Xinghao Ding et al. [8]. The algorithm utilizes color space conversion to obtain a much better visibility. The algorithm has better effectiveness in reducing halo and color distortion.

Yali Feng et al [9] introduced the fast Fourier algorithm into the original algorithm to make the speed faster than that of the conventional method. The method is good for color images but not for gray images.

HT Nguyenl et al. [11], proposed a multilayer feed forward network for the classification of Diabetic Retinopathy. María García et al. [12] used a multilayer perceptron (MLP) classifier to obtain a final segmentation of HEs in the image. In [13] Alireza Osareh et al. classified the segmented regions into two classes, exudates and non-exudates by comparing the performance of various classifiers. Jian Wu et.al [14] proposed a cerebral aneurysm recognition method using Bayesian classification.

In [15], Yosawin Kangwanariyakul et al. proposed to use Back-propagation neural network (BPNN), the Bayesian neural network (BNN), the probabilistic neural network (PNN) and the support vector machine (SVM)for developing classification models for identifying IHD patients. Lili Xu and Shuqian 
Luo in [16] used a support vector machine (SVM) with Gaussian radial basis function as a classifier to identify hard exudates from digital retinal images. In [17], Priya et al. used SVM for the detection of diabetic retinopathy stages using color fundus images. Another work by the same authors [20] implemented artificial neural networks for the prediction of stroke using symptoms and risk factors as the input parameters.

\section{Proposed Method}

The major blood vessels of the retina are radiated from the center of the optic disc. The information of blood vessels, such as length, width, tortuosity and branching pattern can provide information on pathological changes and can automatically diagnose the disease. Optic Disc is normally modeled as a circle. Its radius and center coordinates can be directly selected in the input image. Once the optic disc is located, the user can set several radii of interest centered at the optic disc, where the vessels will be analyzed. The images were cropped using a mask to cover a region of interest corresponding to a circle of 4 OD diameter centered at OD center. [19]

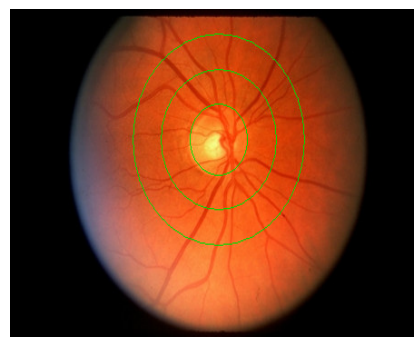

Figure. 1 Detection of Optic Disc and the Zone to be analyzed

Then preprocessing can be implemented to remove the problems of uneven illumination. Multiscale retinex has been implemented in this work for the pre processing of retinal images. Morphological operations are done for the removal of noisy background. For proper vessel segmentation, thresholding can be used to create binary images. To reduce all objects in the vascular map to lines, skeletonization is done. Branching points are detected and parameters like mean diameter, major axis length, eccentricity, fractal dimension and tortuosity are computed. These features have been calculated for healthy retinas, retinas of patients with diabetic retinopathy, hypertensive retinopathy, retinal ischemia and classification is done using Support Vector machine (SVM).

\subsection{Image Acquisition}

A set of 20 images of diabetic Retinopathy acquired using a Canon CR5 non-mydriatic 3CCD camera with a 45 degree field of view (FOV) is taken from DRIVE database [18]. A set of 20 Images of hypertensive retinopathy were taken from VICAVR database [24] which have been acquired with a TopCon non-mydriatic camera NW-100 model .Twenty images of Retinal ischemia has been obtained from Retina Image Bank (ASRS).15 retinal images of healthy persons have been selected both from HRF (High Resolution Fundus) database and DRIVE database.

\subsection{Pre-Processing}

The contrast of medical images is very low and as well as have strong speckle noise. De-noising these speckle noise has become the most important step in medical image processing. The Retinex is a type of preprocessing technique that can be applied to retinal images and is established at the scientific experiments and the scientific analysis. Retinex is a very versatile automatic method which can provide sharpening, color constancy, dynamic range compression and color rendition simultaneously. 

Video Processing, Volume 5 No 2, April (2017); pp: 13-21

In Single Scale Retinex (SSR) instead of applying logarithmic function on the image, the image signal is passed through the Gaussian filter called retinex filter. The output of the retinex filter is used only for scaling the original image signal such that the pixel values are scaled by different weights based on filter output. Logarithmic function is then applied on scaled image. Image filtering using retinex function may require different Gaussian shaped impulse response with different variance. So, MSR (Multiscale Retinex) approach [10] is used.

SSR is mathematically expressed as

$$
R i(x 1, x 2)=\log (\operatorname{Ii}(x 1, x 2))-\log (\operatorname{Ii}(x 1, x 2) * F(x 1, x 2))
$$

F is a Gaussian filter defined by

$$
F(x 1, x 2)=k \exp \left[-\left(x 1^{2}+x 2^{2}\right) / \sigma^{2}\right]
$$

The MSR can be written as

$$
R(x, y)=\sum_{k=1}^{K} \mathrm{~W}_{\mathrm{k}} \cdot\left\{\log [\mathrm{Ii}(x, y)]-\log \left[\operatorname{Ii}(x, y) * \mathrm{~F}_{\mathrm{k}}(x, y)\right]\right\}
$$

Surround function is,

$$
F_{k}(x, y)=k \exp \left[-\frac{x^{2}+y^{2}}{\sigma k^{2}}\right]
$$

The MSR estimates scene reflectance from the ratios of scene intensities to their local intensity averages. The scene is decomposed into a set of images that represent the mean of the image at various spatial resolutions by applying Gaussian filters of different sizes. A set of images that measure the scene reflectance is produced by dividing the original picture point wise by the decomposed picture. A log function is applied to each of the images to reduce the image dynamic range. By adding the compressed images together, the displayed image is finally reconstructed.

Figure. 2 shows the block diagram of multiscale retinex preprocessing.

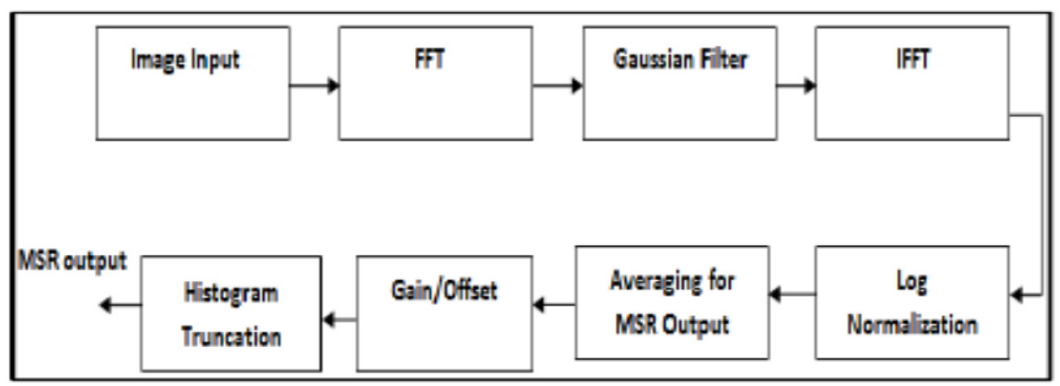

Figure. 2 Block diagram of Multiscale retinex

\subsection{Morphological operations}

The output image obtained after retinex preprocessing is subjected to morphological operations. MATLAB Toolbox function strel constructs structuring elements with a variety of shapes and sizes. The morphological opening function with disc shaped structuring element is applied on this binary image to remove the noisy background.[6] 


\subsection{Binarization}

After removing the background, image is then converted to binary form by applying thresholding. A binary image $B$ can be obtained from a gray scale or color image through an operation that selects a subset of the image pixels as foreground pixels, the pixels of importance in an image analysis task, leaving the rest as background pixels to be ignored. The selection can be as simple as thresholding operation that chooses pixels in a certain range of gray-tones or it may be a complex classification algorithm.

\subsection{Skeletonization}

The structural shape of a plane region can be reduced to a line graph called skeleton. The skeleton of the region can be obtained by a thinning algorithm. It is a way to reduce binary image objects to a set of thin strokes that retain important information about the shape of the original object. After obtaining skeletonized output, the branching points of skeleton are detected and the lengths of branches are calculated.

\subsection{Feature Extraction}

After performing the above operations, the features of the processed image need to be extracted. MATLAB function bwdistgeodesic computes the geodesic distance transform of the binary image. A set of properties for each connected component in the binary image are computed. They include parameters [7] like eccentricity, orientation and major axis length .Mean diameter, tortuosity and fractal dimension has also been evaluated. Number of branching points can also be computed from the skeletonized image.

\subsubsection{Mean Diameter:}

An estimate of vessel diameters is calculated using the distance transform of the inverted binary preprocessed image. This gives the Euclidean distance of every 'vessel' pixel from the closest nonvessel pixel, and therefore doubling the maximum value of the distance transform along the thinned centrelines provides an estimate of the diameter of every vessel segment at its widest point [22]. Then, the mean diameter is computed.

\subsubsection{Tortuosity:}

The clinical recognition of abnormal retinal tortuosity is significant in the diagnosis of several ocular and systemic diseases. Tortuosity is best described as the meanderness of the vessels, and engorgement in the diameter of the vessel. This method employs the basic idea of numerical integration [21] to determine the value of curvature for the retinal vessel curves from the detected blood vessels. The curvature value can be calculated by the ratio of the path length $(L)$ and the shortest path (C).

\subsubsection{Fractal Dimension}

Fractal analysis could be applied in automated diagnosis of retinal diseases since retinal vessels are fractal. Fractal dimension [19] is a complexity indicator determined by reactive vessels obtained from vascular tracking through the box counting dimension method. After vessel tracing was ascertained, $\mathrm{D}_{f}$ is computed from the refined skeletonized vessel tracing using the Box-Counting method. The box counting equation briefly is:

$$
D_{f}=\lim _{r \rightarrow 0} \log (N[r]) / \log \left(\frac{1}{r}\right)
$$


Where $N(r)$ is the number of boxes overlying a fractal structure and $r$ is the side length of each box.

\subsection{Classification}

This work focuses on the development of an artificial neural network model with 6 input parameters and 4 output classes for the prediction of retinal eye disease. Special importance is given to the prediction of retinal ischemia as it is an initial symptom for the occurance of stroke. The number of input nodes are determined by the finalized input data, the number of hidden nodes are determined through trial and error method and the output nodes determines the number of output classes. Here, a multilayered feed forward neural network has been designed with 6 input nodes, a hidden layer with 8 hidden nodes and 4 output nodes. Backpropogation algorithm [23] with sigmoid activation function is used to train the feed forward neural network architecture.

\section{Results \& discussion}

The various output stages before feature extraction is shown in Fig. 3

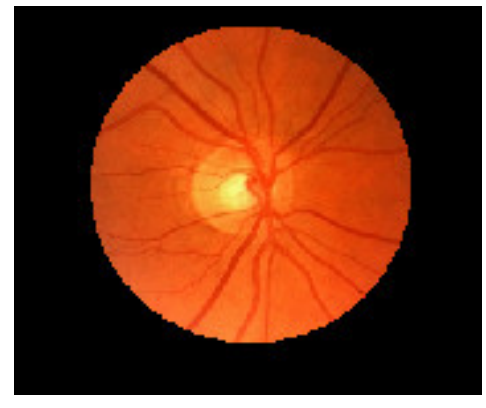

(a)

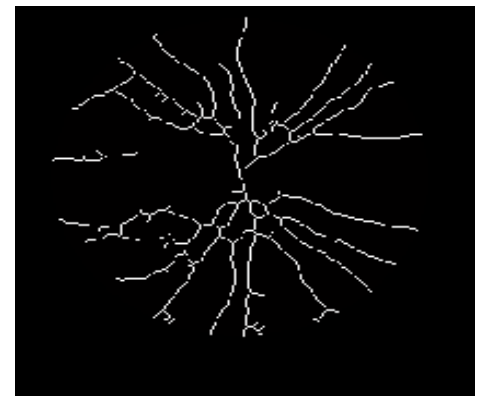

(d)

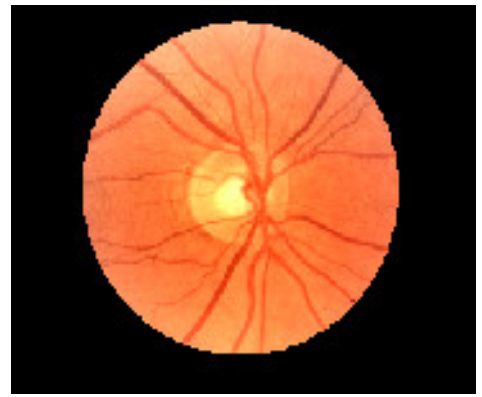

(b)

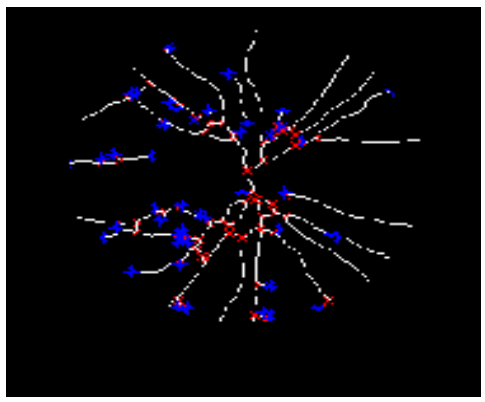

(e)

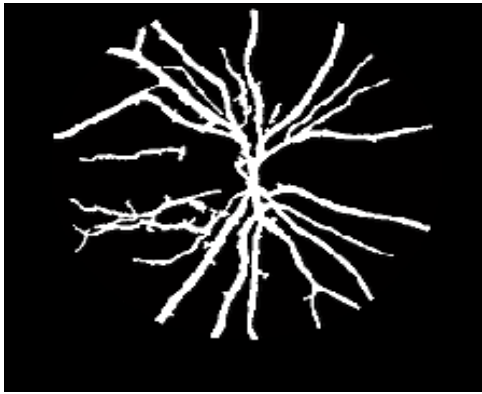

(c)

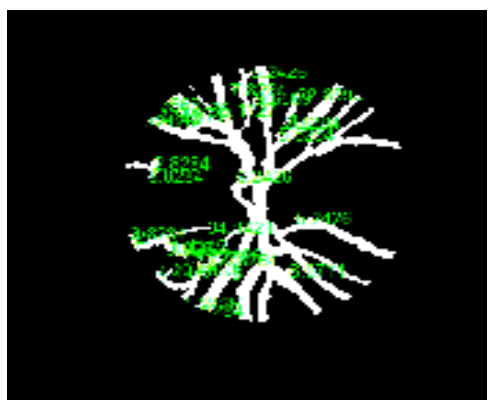

(f)

Figure. 3 Various Output Stages of a healthy retinal fundus image

Cropped healthy retinal image (b) Retinex preprocessed image (c) Binarized image

(d) Skeletonized image (e) Detection of branch points and end points ( $f$ ) Computing length of branches

The all dataset have been divided into 3 classes. Using the training dataset, the recognition accuracy is $100 \%$ and using (i) testing dataset of 22 samples, the accuracy is $89 \%$

ii) testing dataset of 37 samples, the accuracy is $85 \%$. 
Table 1 shows the recognition accuracy for various training and testing datasets.

Table 1. Accuracy for various datasets

\begin{tabular}{|c|c|c|}
\hline $\begin{array}{c}\text { Number of patterns in } \\
\text { training dataset }\end{array}$ & $\begin{array}{c}\text { Number of patterns in } \\
\text { testing dataset }\end{array}$ & $\begin{array}{c}\text { Recognition } \\
\text { Accuracy }\end{array}$ \\
\hline 53 & 22 & $89 \%$ \\
\hline 50 & 50 ( same training set) & $100 \%$ \\
\hline 37 & 37 & $85 \%$ \\
\hline
\end{tabular}

The performance plot of ANN with backpropogation is shown in Fig.4

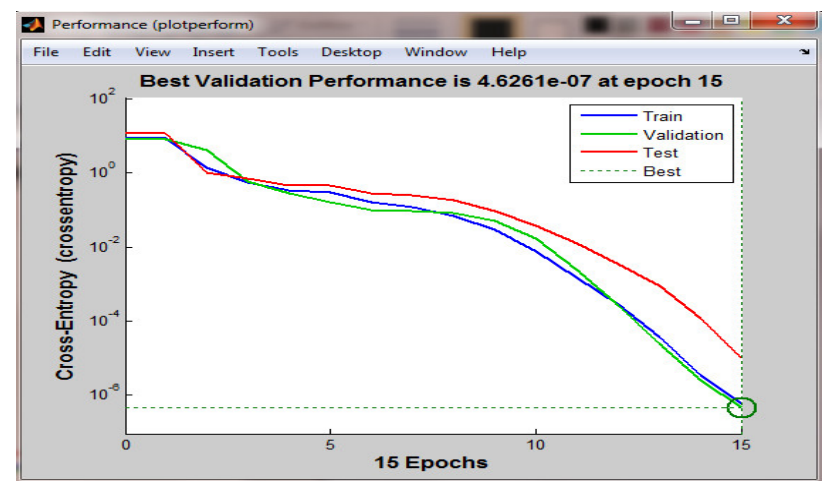

Figure . 4 Performance plot of ANN

Validation requires the calculation of statistical parameters like sensitivity, accuracy, precision and F1 score. The ability of the method to identify correct cases is given by Sensitivity. The fraction of correct classifications to the total number of classifications is given by accuracy. Precision is the likelihood that a retrieved case is relevant. F1 Score gives the harmonic mean of precision and recall (sensitivity).

Table 2 shows the statistical parameters evaluated for an ANN classifier.

Table 1. Evaluation of statistical parameters

\begin{tabular}{|c|c|c|c|}
\hline Accuracy & Precision & Sensitivity & F1 Score \\
\hline $89 \%$ & $100 \%$ & $89 \%$ & $94 \%$ \\
\hline
\end{tabular}

Observation shows that ANN can be used in predicting the probability of eye diseases as it was able to give an accuracy of $89 \%$. Retinal ischemia proves to be an important biomarker of stroke and so special significance should be given in the prediction of retinal ischemia as it can predict the possibility of occurrence of stroke.

\section{Conclusion}

A neural network model developed in this work for the prediction of eye diseases was able to achieve an accuracy of $89 \%$. Retinal imaging aids in predicting the probability of stroke based on parameters evaluated from the vascular map of retinal ischemia. Early detection of cardiovascular diseases like stroke through biomarkers derived from retinal imaging would allow patients to be treated more effectively. Performance of the system can be improved by incorporating more features like A/V ratio of the branching vessels and requires training from a much larger database. Interdisciplinary teams 
Jeena R S, Dr. Sukesh Kumar A; Stroke Prognosis through Retinal Image Analysis. Advances in Image and Video Processing, Volume 5 No 2, April (2017); pp: 13-21

will be able to explore the interface at the border between ophthalmology, neurology and computing science .Since the microvasculature of brain and eye are interlinked, it can be confirmed that in vivo retinal neurovascular measures are predictive of microvascular change in the brain .

\section{REFERENCES}

[1] Barry L. Zaret, M.D., Marvin Moser, M.D., Lawrence S. Cohen, Chapter 18 Stroke - Lawrence M. Brass, M.D. (pgs 215-234)

[2] Baker ML,Wang JJ,Liew G et al,Differential associations of cortical and subcortical cerebral atropy with retinal vascular signs in patients with acute stroke,Stroke41,2143-50

[3] E. Land (1986).An alternative technique for the computation of the designator in the retinex theory of color vision, in Proc. Nat. Acad. Sci., vol. 83, pp.3078-3080.

[4] D. J. Jobson, Z. Rahman, and G. A. Woodell (1997). A Multiscale retinex for bridging the gap between color images and the human observation of scenes, IEEE Transaction Image Processing, Vol. 6, No. 7, pp. 965-976, July.

[5] Youhei Terai, TomioGoto, Satoshi Hirano, and Masaru Sakurai (2009). Color Image Contrast Enhancement by Retinex Model, Proceedings of IEEE $13^{\text {th }}$ International Symposium on Consumer Electronics, pp. 392-393, May.

[6] Manjiri B. Patwari, Ramesh R. Manza, Yogesh M. Rajput, Deepali D. Rathod, Manoj Saswade, Neha Deshpande,"Classification and Calculation of Retinal Blood vessels Parameters", IEEE's INTERNATIONAL CONFERENCES FOR CONVERGENCE OF TECHNOLOGY, Pune,India.

[7] Patwari Manjiri, Manza Ramesh, Rajput Yogesh, Saswade Manoj, Deshpande Neha, “Automated Localization of Optic Disk, Detection of Microaneurysms and Extraction of Blood Vessels to Bypass Angiography", Springer, Advances in Intelligent Systems and Computing. ISBN: 978-3-319-11933-5, DOI: 10.1007/978-3-319-11933-5_65. 2014

[8] Yali Feng, Jing Huang, Zhuoli Feng and Minyong Liu (2011). The Research and Implementation of Light Compensation Algorithm in Color Facial Image, Electrical and Control Engineering Conference,pp.2758-276.

[9] Mrs. Anjali Chandra, BibhudendraAcharya \&Mohammad Imroze Khan (2011). Retinex image processing: Improving the visual realism of color images, International Journal of Information Technology and Knowledge Management, Volume 4, No. 2, pp. 371-377

[10] Priyanka J K,Dr B G Sudarshanan,Dr S C Prasanna Kumar,Dr N Pradhan,"Development of algorithm for high resolution retinex for image enhancement", International journal of Innovative Research development,December 2012.

[11] HT Nguyen, M Butler, A Roychoudhry, AG Shannon, J Flack, P Mitchell, -Classification of Diabetic retinopathy using neural networks , 18th Annual International Conference of the IEEE Engineering in Medicine and Biology Society, Amsterdam 1996. 
[12] María García, Roberto Hornero, Clara I. Sanchez, María I. Lopez and Ana Díez, -Feature Extraction and Selection for the Automatic Detection of Hard Exudates in Retinal Images, Proceedings of the 29th Annual International Conference of the IEEE EMBS Cite Internationale, Lyon, France, August 2326, 2007.

[13] Alireza Osareh, Majid Mirmehdi, Barry Thomas, and Richard Markham, -Classification and Localisation of Diabetic-Related Eye Disease , Springer-Verlag Berlin Heidelberg ,pp. 502- 516, 2002

[14] Jian Wu, Guangming Zhang, Yanyan Cao, and Zhiming Cui, Research on Cerebral Aneurysm Image Recognition Method Using Bayesian Classification -, Proceedings of the 2009 International Symposium on Information Processing (ISIP'09), Huangshan, P. R. China, August 21-23, pp. 058-062, 2009.

[15] Yosawin Kangwanariyakul, Chanin Nantasenamat, Tanawut Tantimongcolwat, Thanaokorn Naenna, Data Mining Of Magnetocardiograms For Prediction of Ischemic Heart Disease EXCLI Journal, 2010.

[16] Lili Xu, Shuqian Luo, Support Vector Machine Based Method For Identifying Hard Exudates In Retinal Images, IEEE, 2009.

[17] Priya.R , Aruna.P, Automated Classification System For Early Detection Of Diabetic Retinopathy In Fundus Images, International Journal Of Applied Engineering Research, Dindigul, Volume 1, No 3,2010 .

[18] J.J. Staal, M.D. Abramoff, M. Niemeijer, M.A. Viergever, B. van Ginneken, "Ridge based vessel segmentation in color images of the retina", IEEE Transactions on Medical Imaging, 2004, vol. 23, pp. 501-509.

[19] Behzad Aliahmad, D K Kumar, Hao Hao, Premith Unnikrishnan, M.Z Che Azemin, R Kawasaki, P Mitchell,Zone Specific fractal dimension of Retinal images as Predictor of Stroke Incidence, Hindawi Publication, Volume 2014

[20] Jeena R S,Dr SukeshKumar A,'Artificial Neural Networks in Stroke Prediction', International Conference on Innovative Systems, December 2016,Bangalore

[21] Grisan E, Foracchia M, Ruggeri A (2008) A novel method for the automatic grading of retinal vessel tortuosity. IEEE Trans Med Imag 27, 310-9

[22] Bankhead P, Scholfield CN, McGeown JG, Curtis TM (2012) Fast Retinal Vessel Detection and Measurement Using Wavelets and Edge Location Refinement. PLoS ONE 7(3): e32435. doi:10.1371/journal.pone.0032435

[23] Yu, Chien-Cheng, and Bin-Da Liu. "A backpropagation algorithm with adaptive learning rate and momentum coefficient." Neural Networks, 2002. IJCNN'02. Proceedings of the 2002 International Joint Conference on. Vol. 2. IEEE, 2002.

[24] S. G. Vázquez, N. Barreira, M. G. Penedo, M. Ortega, A. Pose-Reino, "Improvements in Retinal Vessel Clustering Techniques:Towards the Automatic Computation of the Arterio Venous Ratio", Computing, Archives for Scientific Computing, 90 (3), 197-217, 2010. 